PROCEEDINGS OF THE

AMERICAN MATHEMATICAL SOCIETY

Volume 125, Number 9, September 1997, Pages 2551-2553

S 0002-9939(97)04056-

\title{
THE $K$-THEORY OF GROMOV'S TRANSLATION ALGEBRAS AND THE AMENABILITY OF DISCRETE GROUPS
}

\author{
GÁBOR ELEK
}

(Communicated by Palle E. T. Jorgensen)

\begin{abstract}
We prove the following theorem. A finitely generated group $\Gamma$ is amenable if and only if $\mathbf{1} \neq \mathbf{0}$ in $K_{0}(T(\Gamma))$, the algebraic $K$-theory group of its translation algebra.
\end{abstract}

\section{INTRODUCTION}

The translation algebras of finitely generated groups were introduced by Gromov ([3], page 262). Let $\Gamma$ be a finitely generated group and let $C_{\Gamma}$ be a Cayley-graph of $\Gamma$. Then $\Gamma$ can be equipped with the shortest distance metric, dist $: \Gamma \times \Gamma \rightarrow \mathbf{R}$. A matrix of finite propagation is a function $A: \Gamma \times \Gamma \rightarrow \mathbf{R}$ with the following properties. There exist positive constants $k_{A}, m_{A}$ such that for any $(\gamma, \delta) \in \Gamma \times \Gamma$ : 1. $|A(\gamma, \delta)| \leq m_{A}$, 2. $A(\gamma, \delta)=0$, if $\operatorname{dist}(\gamma, \delta)>k_{A}$.

The matrices of finite propagation form the translation algebra of $\Gamma$, which we denote by $T(\Gamma)$. (Note that $T(\Gamma)$ does not depend on the choice of the Cayleygraph $C_{\Gamma}$.) The goal of this paper is to prove the following theorem.

Theorem 1. Let $\Gamma$ be a finitely generated group. Then $\Gamma$ is amenable if and only if $\mathbf{1} \neq \mathbf{0}$ in $K_{0}(T(\Gamma))$, the algebraic $K$-theory group of $T(\Gamma)$.

The reader should note that our theorem can be regarded as an analog of Theorem 3.1 in [1].

\section{Amenable groups}

Let $\Gamma$ be a finitely generated amenable group. Then the following proposition holds.

Proposition 2.1. There exists a finite trace $\operatorname{Tr}_{\omega}$ on $T(\Gamma)$, such that $\operatorname{Tr}_{\omega}(\mathbf{1})=1$ and $\operatorname{Tr}_{\omega}(\mathbf{0})=0$.

Note that the proposition is a combinatorial analogue of a result of John Roe [4]. Finite traces can be extended to the algebraic $K$-theory group; hence we have the following corollary.

Proposition 2.2. Let $\Gamma$ be as above. Then $\mathbf{1}$ and $\mathbf{0}$ are not representing the same element in $K_{0}(T(\Gamma))$.

Received by the editors April 9, 1996.

1991 Mathematics Subject Classification. Primary 20F38.

(C)1997 American Mathematical Society 
Proof of Proposition 2.1. Let $\omega$ be a Banach-mean (ultralimit) on the bounded, infinite real sequences $\mathbf{a}=\left(a_{1}, a_{2}, \ldots\right)$, such that $\omega(\mathbf{a})=\lim _{n \rightarrow \infty} a_{n}$ if $\lim _{n \rightarrow \infty} a_{n}$ exists. Again, let us consider a Cayley-graph of $\Gamma$ and the induced metric structure on the group. The amenability of $\Gamma$ is characterized by the following F $\varnothing$ lner-property [5].

Let $B_{n}=\{y \in \Gamma \mid \operatorname{dist}(y, e) \leq n\}$, the $n$-ball centered at the identity element of $\Gamma$. Then, for any $k>0$,

$$
\lim _{n \rightarrow \infty} \frac{\#\left(B_{n+k} \backslash B_{n}\right)}{\#\left(B_{n}\right)}=0
$$

We define $\operatorname{Tr}_{\omega}$ as follows. Let $A \in T(\Gamma)$; then $\operatorname{Tr}_{\omega}(A)=\omega(\mathbf{a})$, where

$$
a_{n}=\frac{1}{\#\left(B_{n}\right)} \sum_{x \in B_{n}} A(x, x) \text {. }
$$

Obviously, $\operatorname{Tr}_{\omega}(\mathbf{1})=1$ and $\operatorname{Tr}_{\omega}(\mathbf{0})=0$. Hence the only thing that needs to be shown is that, for any $R, S \in T(\Gamma), \operatorname{Tr}_{\omega}(R S)=\operatorname{Tr}_{\omega}(S R)$.

For $A \in T(\Gamma), n>0$, let $A_{n}$ be a $\#\left(B_{n}\right) \times \#\left(B_{n}\right)$-matrix with entries parametrized by pairs of vertices of $B_{n}$, such that $\left(A_{n}\right)_{i, j}=A(i, j)$, if $i, j \in B_{n}$.

Lemma 2.3.

$$
\lim _{n \rightarrow \infty} \frac{\operatorname{Tr}(R S)_{n}-\operatorname{Tr}\left(R_{n} S_{n}\right)}{\#\left(B_{n}\right)}=0
$$

Proof.

$$
\begin{gathered}
\operatorname{Tr}(R S)_{n}=\sum_{i \in B_{n}} \sum_{j \in \Gamma} R(i, j) S(j, i) \\
=\operatorname{Tr}\left(R_{n} S_{n}\right)+\sum_{i \in B_{n}} \sum_{j \in B_{n+k_{S}} \backslash B_{n}} R(i, j) S(j, i)
\end{gathered}
$$

by the finite propagation property of $S$. However, $R(i, j) S(j, i)=0$, if $\operatorname{dist}(i, j)>$ $k_{R}$. Therefore, we have the following estimate.

$$
\operatorname{Tr}\left(R_{n} S_{n}\right)+\sum_{i \in B_{n}} \sum_{j \in B_{n+k_{S}} \backslash B_{n}} R(i, j) S(j, i) \leq m_{R} m_{S} \#\left(B_{k_{R}}\right) \#\left(B_{n+k_{S}} \backslash B_{n}\right) .
$$

That is,

$$
\left|\frac{\operatorname{Tr}(R S)_{n}-\operatorname{Tr}\left(R_{n} S_{n}\right)}{\#\left(B_{n}\right)}\right| \leq \frac{m_{R} m_{S} \#\left(B_{k_{R}}\right) \#\left(B_{n+k_{S}} \backslash B_{n}\right)}{\#\left(B_{n}\right)} .
$$

The right-hand side of the inequality above tends to zero by the Følner-property. This proves our lemma.

Now we return to the proof of Proposition 2.1. By our definition, $\operatorname{Tr}_{\omega}(R S)=$ $\omega(\mathbf{x})$, where $x_{n}=\frac{1}{\#\left(B_{n}\right)} \operatorname{Tr}(R S)_{n}$. Hence by our previous lemma,

$$
\operatorname{Tr}_{\omega}(R S)=\omega(\mathbf{y}),
$$

where $y_{n}=\frac{1}{\#\left(B_{n}\right)} \operatorname{Tr}\left(R_{n} S_{n}\right)$. On the other hand, $\operatorname{Tr}_{\omega}(S R)=\omega(\mathbf{z})$, where $z_{n}=$ $\frac{1}{\#\left(B_{n}\right)} \operatorname{Tr}(S R)_{n}$. Thus,

$$
\operatorname{Tr}_{\omega}(S R)=\omega(\mathbf{w}),
$$

where $w_{n}=\frac{1}{\#\left(B_{n}\right)} \operatorname{Tr}\left(S_{n} R_{n}\right)$. However, $y_{n}=w_{n}$, so $\operatorname{Tr}_{\omega}(R S)=\operatorname{Tr}_{\omega}(S R)$. 


\section{NON-AMENABLE GROUPS}

First let us review a result of Deuber, Simonovits and Sós on paradoxical metric spaces [2].

Let $X$ be a metric space. Then $Y \subset X$ is wobbling equivalent to $X$, if there exists a bijection $\phi: X \rightarrow Y$, such that

$$
\sup _{x \in X} \operatorname{dist}(\phi(x), x)<\infty
$$

According to Theorem 3.1 [2], if $X$ is a vertex space of a graph with exponential growth, then $X$ can be written as the disjoint union of $Y_{1}$ and $Y_{2}$, where both $Y_{1}$ and $Y_{2}$ are wobbling-equivalent to $X$. Now let $\Gamma$ be a finitely generated non-amenable group. Then $\Gamma$ has exponential growth [5]. Hence we have the partition of $\Gamma$ into $\Gamma_{1} \cup \Gamma_{2}$, where $\phi_{1}: \Gamma \rightarrow \Gamma_{1}$ and $\phi_{2}: \Gamma \rightarrow \Gamma_{2}$ are bijections such that

$$
\sup _{x \in X} \operatorname{dist}\left(\phi_{1}(x), x\right)<\infty \text { and } \sup _{x \in X} \operatorname{dist}\left(\phi_{2}(x), x\right)<\infty \text {. }
$$

Let $U$ be a co-isometry defined the following way. For each $\gamma \in \Gamma, U\left(e_{\gamma}\right)=e_{\phi_{1}(\gamma)}$, where $e_{\gamma}$ is the characteristic function of $\gamma$. Then $U^{*} U=1, U U^{*}=P$, where the idempotent $P$ is the multiplication by the characteristic function of $\Gamma_{1}$. Note that both $U$ and $U^{*}$ are elements of the translation algebra; consequently $\mathbf{1}=P$ in $K_{0}(T(\Gamma))$. The same way, $\mathbf{1}=Q$ in $K_{0}(T(\Gamma))$, where $Q=1-P$, the multiplication by the characteristic function of $\Gamma_{2}$. Therefore, $\mathbf{1}+\mathbf{1}=\mathbf{1}$ in $K_{0}(T(\Gamma))$, which shows the remaining part of Theorem 1.

\section{REFERENCES}

1. J. Block, S. Weinberger, Aperiodic tilings, positive scalar curvature and amenability of spaces, Journal of the Amer. Math. Soc. 5 (1992), 907-918 MR 93d:53054

2. W.A.Deuber,M.Simonovits and V.T.Sós, A note on paradoxical metric spaces, Studia Sci.Hung.Math. 30 (1995), 17-23 MR 96i:54025

3. M.Gromov, Asymptotic Invariants of Infinite Groups, London Math. Society, Lecture Note Series 182 (1993) MR 95m:20041

4. J. Roe, An index theorem on open manifolds I-II, Journal of Differential Geometry 27 (1988), 87-136 MR 89a:58102

5. P.M.Soardi, Potential Theory on Infinite Networks, Lecture Notes in Mathematics $\mathbf{1 5 9 0}$ MR 96i:31005

Department of Mathematics, Purdue University, West Lafayette, Indiana 47906

E-mail address: elekgab@math.purdue.edu

Current address: Mathematical Institute, Hungarian Academy of Science, P. O. Box 127, H-1364 Budapest, Hungary

E-mail address: elek@math-inst.hu 